\title{
Single-Step Procedure for the Isolation of Proteins at Near-Native Conditions from Mammalian Tissue for Proteomic Analysis on Antibody Microarrays
}

\author{
Mohamed Saiel Saeed Alhamdani, ${ }^{*, \dagger}$ Christoph Schröder, ${ }^{\dagger}$ Jens Werner, ${ }^{\ddagger}$ Nathalia Giese, ${ }^{\ddagger}$ \\ Andrea Bauer, ${ }^{\dagger}$ and Jörg D. Hoheisel ${ }^{\dagger}$ \\ Division of Functional Genome Analysis, Deutsches Krebsforschungszentrum (DKFZ), Im Neuenheimer Feld \\ 580, 69120 Heidelberg, Germany, and Department of General Surgery, University of Heidelberg, Im \\ Neuenheimer Feld 110, 69120 Heidelberg, Germany
}

Received September 18, 2009

\begin{abstract}
The process of extracting comprehensive proteome representations is a crucial step for many proteomic studies. While antibody microarrays are an evolving and promising methodology in proteomics, the issue of protein extraction from tissues for this kind of analysis has never been addressed. Here, we describe a single-step extraction buffer for the isolation of proteins from mammalian tissues under native conditions in an effective and reproducible manner. Protein was extracted from cell lines BxPC-3 and SU.86.86, rat organs (pancreas, liver, heart and lung) and human pancreatic cancer tissues using several buffer systems that contained individual nonionic or zwitterionic detergents in comparison to commercial extraction buffers. Also, detergent combinations were used that included at least one polymeric phenylethylene glycol, a long-chain amidosulfobetaine, cholate and a zwitterionic detergent. Extracts were analyzed for protein quantity and quality. The detergent cocktails exhibited superior extraction capacity. Additionally, they demonstrated a substantially higher recovery of membrane and compartmental proteins as well as much better preservation of protein functionality. Also, they did not interfere with subsequent analysis steps such as labeling. In Western blot and antibody microarray assays, they outperformed the other buffer systems, indicating that they should also be useful for other types of proteomic studies.
\end{abstract}

Keywords: Antibody microarray • protein extraction • detergents • compartmentalized protein

\section{Introduction}

Among the advanced methodologies that are being developed for analyzing the proteome, antibody microarrays emerge as a promising tool for gathering information at a global level that is required for the detection of disease-relevant variations, monitoring of disease progression and a better understanding of disease biology. ${ }^{1,2}$ Antibody microarrays are an intrinsically robust and semiquantitative system that performs parallel measurements on sets of known proteins at a high-throughput., By direct labeling of protein samples or label-free detection, the analysis is scalable to nearly any complexity. As in all kinds of proteomic studies, extraction and solubilization of proteins is a critical factor for the experimental outcome. ${ }^{5}$ Owing to the complexity of the proteome, the enormous dynamic range in concentration, compartmentalization of proteins within cells and tissues as well as the susceptibility of proteins to minimal changes in the milieu and their relative abundance, an appropriate processing of a sample for proteomic analysis is a

\footnotetext{
* Corresponding author: Mohamed Saiel Saeed Alhamdani, Functional Genome Analysis, Deutsches Krebsforschungszentrum, Im Neuenheimer Feld 580, D-69120 Heidelberg, Germany. Tel.: [49] (6221) 42-4684. Fax: [49] (6221) 42-4687. E-mail: m.alhamdani@dkfz.de.

${ }^{\dagger}$ Deutsches Krebsforschungszentrum (DKFZ).

₹ University of Heidelberg.
}

challenging task. The majority of recent antibody microarray studies analyzed serum samples. However, other types of specimens were investigated as well, such as extracts of cell surface proteins, ${ }^{6}$ cultured cells ${ }^{7}$ or tissue biopsies. ${ }^{8}$ Proteins were mostly isolated by procedures adapted from immunoblotting or enzyme-linked immunosorbent assay (ELISA), or extraction buffers from commercial sources were used. However, buffers for immunoblotting or ELISA are aiming at the extraction of certain proteins or a group of proteins rather than a full representation of the cellular proteome. Commercial buffers, on the other hand, are frequently unsatisfactory due to a rather limited extraction capacity and/or difficulties in identifying the source of error in downstream analysis owing to the unknown nature of their formulation.

We are involved in studies of global expression variations by means of antibody microarray analysis. To this end, we established the processes for production, incubation and target labeling. ${ }^{9-11}$ However, protein preparation remained a critical and complicated factor, especially for the analysis of tissues. Optimized procedures for isolating and solubilizing proteins for gel-based studies ${ }^{12}$ or mass spectrometry ${ }^{13}$ were found inapplicable to antibody microarrays. On the basis of recent experiences made for the isolation of protein fractions, ${ }^{14}$ we 
looked into processes for isolating proteins in a comprehensive and simultaneously gentle manner, which deliver proteins in a near-native state. In addition, we aimed at a simple and highly reproducible process. Detergent-based protein extraction is one of the easiest, highly efficient and least harsh methods for isolating proteins from biological samples. ${ }^{15}$ However, no zwitterionic or nonionic detergent and no detergent mixture was reported to solubilize all proteins. ${ }^{5}$ Detergent selection for solubilization conditions has been an empirical and experimental process for a given set of samples. ${ }^{16}$ Using a combination of detergents, which adds to the mixture the best properties of each, is likely to be more effective than using detergents individually. ${ }^{17}$ Here, we describe a protein extraction formula for a one-step isolation of proteins from cell culture or mammalian tissues that is highly reproducible and effective as well as compatible with analysis on antibody microarrays. The buffer extracts proteins under near-native conditions and achieves an enrichment of membrane-associated and compartmentalized proteins.

\section{Materials and Methods}

Materials. All chemicals used in this study were purchased from Sigma-Aldrich unless otherwise stated and were of highest purity or protein grade. Thirty-eight polyclonal and monoclonal antibodies were purchased from different commercial sources and used for printing the test array. Ten of these antibodies were also used in the Western blot experiments.

Buffer Formulation. The basic buffer composition was formulated after several tests. It consists of $20 \mathrm{mM}$ HEPES buffer, pH 7.9, 1 mM MgCl 2,5 mM EDTA, 1 mM PMSF, $1 \mathrm{U} / \mu \mathrm{L}$ of Benzonase, $1 \times$ Halt Protease and Phosphatase Inhibitor Cocktail (Thermo Scientific, Bonn, Germany) and detergent(s). When detergents were added individually, their final concentration was $1 \%(\mathrm{w} / \mathrm{v})$ for Nonidet P-40 substitute (NP-40S), Triton X100 (TrX100) and cholic acid sodium salt (Cholate) or 0.5\% (w/v) for 3-[(3-cholamidopropyl)dimethylammonio]-1propanesulfonate (CHAPS) (Serva, Heidelberg, Germany), 3-(4heptyl)phenyl-3-hydroxypropyl) dimethyl-ammoniopropanesulfonate (C7BzO), amidosulfobetaine-14 (ASB-14), $n$-dodecyl$\beta$-D-maltoside (12-Malt) (GenaXXon Bioscience GmbH, Ulm, Germany) and $n$-octyl- $\beta$-D-glucoside (8-Glu). Also, two detergent cocktails were used: Mixl was composed of the basic buffer and $0.5 \%$ TrX100, 0.5\% NP-40S, 0.25\% 12-Malt, $0.25 \%$ ASB-14 and $0.25 \%$ CHAPS; Mix2 contained as detergents $1 \%$ NP-40S, $0.5 \%$ cholate, $0.25 \%$-Glu and $0.25 \%$ ASB- 14 . In addition, several commercial buffers were tested for their protein extraction efficiency. Because of their superior performance within the group of commercial buffers, the Q-Proteome Mammalian Protein Prep Kit (Q-Prot; Qiagen, Hilden, Germany) and the Mammalian Protein Extraction Reagent (M-PER; Thermo Scientific) were selected for a more detailed comparison.

Protein Extraction from Cell Culture. The pancreatic cancer cell lines BxPC-3 and SU.86.86 were used in this experiment. The cells were cultured in RPMI-1640 medium supplemented with $10 \%$ fetal calf serum and antibiotics at $37^{\circ} \mathrm{C}$. At $90 \%$ confluence, cells were collected by trypsination, washed three times with ice-cold PBS, lysed with the respective extraction buffer (10-times the volume of the packed cells), kept on ice for $30 \mathrm{~min}$ with occasional mixing, and then centrifuged at $20000 \mathrm{~g}$ for $20 \mathrm{~min}$ at $4{ }^{\circ} \mathrm{C}$. The supernatant was aspirated with a fine needle in order not to disturb the upper layer or the pellet. Protein concentration in the supernatant was deter- mined with the bicinchoninic acid (BCA) Protein Assay Reagent kit of Thermo Scientific (Bonn, Germany).

Protein Extraction from Tissues. Liver, heart, lung and pancreatic tissues were dissected under sterile conditions from adult male Wistar rats and stored at $-80{ }^{\circ} \mathrm{C}$ until further processing. The animals were kept in accordance with the institutional and national guidelines. Twelve human pancreatic cancer tissue samples were selected randomly from a set of about 400 samples provided by the Surgery Department of the University of Heidelberg. Written informed consent was obtained from all patients and the local ethics committee of the University of Heidelberg approved the work.

Tissue samples were either immediately homogenized in 5 -fold by weight the amount of extraction buffer with the aid of an Ultra Turrax homogenizer (IKA Werke GmbH, Staufen, Germany), a Dounce or a Potter-Elvehjem homogenizer, respectively. Alternatively, tissues were snap-frozen in liquid nitrogen followed by pulverization with a porcelain mortar and pestle in presence of liquid nitrogen and immediately resuspended in 5-fold by weight the amount of extraction buffer. Homogenates were incubated on ice for $30 \mathrm{~min}$ with occasional mixing and processed the same way as the cell culture samples.

Enzymatic Activity Assays. All enzymatic analyses were performed with unlabeled protein. Labeling of the protein samples with fluorescent dyes for incubation on the antibody microarrays may well have an effect on protein functions.

1. Gamma-glutamyltranspeptidase ( $\gamma$-GT) Activity Assay. The activity of membrane bound enzyme $\gamma$-GT was assessed in protein extracts according to the procedure of Shaw et al. ${ }^{18}$ In brief, the reaction mixture contained $4.4 \mathrm{mM} \gamma$-glutamyl$p$-nitroanilide, $20 \mathrm{mM}$ glycylglycine, $11 \mathrm{mM} \mathrm{MgCl}_{2}$ and $100 \mathrm{mM}$ Tris-HCl buffer $(\mathrm{pH} 7.9)$ in a final volume of $100 \mu \mathrm{L}$. The reaction was started by the addition of $20 \mu \mathrm{L}$ of sample extract to the reaction mixture in a 96 -well plate. The change in absorbance was monitored at $405 \mathrm{~nm}$ and $25^{\circ} \mathrm{C}$ for $5 \mathrm{~min}$ in a Tecan Infinite-200 plate reader (Crailsheim, Germany). The activity was calculated from the extinction coefficient of p-nitroanilide $\left(9900 \mathrm{M}^{-1} \mathrm{~cm}^{-1}\right)$ and expressed as international units per milligram of protein (IU/mg protein).

2. Glutathione $S$-transferase (GST) Activity Assay. The total activity of GST (cytoplasmic and membrane bound) was determined in extracted protein samples as described previously, ${ }^{19}$ with the modification of using 1-chloro-2,4-dinitrobenzene (CDNB) as substrate. The reaction mixture was composed of $1.25 \mathrm{mM}$ reduced glutathione, $1 \mathrm{mM} \mathrm{CDNB}, 1 \%$ ethanol and $100 \mathrm{mM}$ potassium phosphate buffer, $\mathrm{pH}$ 6.5. For each sample, $5 \mu \mathrm{L}$ was transferred into a well of a 96 -well plate and the reaction was initiated by adding $215 \mu \mathrm{L}$ of the reaction mixture. The increase in absorbance was followed at $340 \mathrm{~nm}$ and $25^{\circ} \mathrm{C}$ for $10 \mathrm{~min}$ using the Tecan Infinite-200 plate reader. The activity was calculated from the extinction coefficient of 9600 $\mathrm{M}^{-1} \mathrm{~cm}^{-1}$ for CDNB.

3. Lactate Dehydrogenase (LDH) Activity Assay. LDH was assayed according to Babson and Babson. ${ }^{20}$ Briefly, a color reagent was prepared by dissolving $40 \mathrm{mg}$ of 2 - $p$-iodophenyl3- $p$-nitrophenyl-5-phenyl tetrazolium chloride (INT), $100 \mathrm{mg}$ of $\beta$-nicotinamide adenine dinucleotide oxidized (NAD) and $10 \mathrm{mg}$ of phenazine methosulfate (PMS) in $20 \mathrm{~mL}$ of water. A sample volume of $20 \mu \mathrm{L}$ was transferred into a well of a 96well plate followed by the addition of $200 \mu \mathrm{L}$ of substrate solution containing $50 \mathrm{mM}$ lactate in $100 \mathrm{mM}$ Tris- $\mathrm{HCl}$ buffer, $\mathrm{pH}$ 8.2. The temperature was adjusted to $30^{\circ} \mathrm{C}$. Then, $40 \mu \mathrm{L}$ of the color reagent was added and the increase in absorbance 
was monitored at $503 \mathrm{~nm}$ for 2 min using the Tecan Infinite200 plate reader. $\mathrm{LDH}$ specific activity was calculated from the extinction coefficient of INT (19 $\left.300 \mathrm{M}^{-1} \mathrm{~cm}^{-1}\right)$.

SDS-PAGE and Western Immunoblot Analysis. Samples of protein extracts were diluted in $2 \times$ Laemmli buffer $(0.125 \mathrm{M}$ Tris-HCl, pH 6.8, 4\% SDS, $20 \%$ glycerol, $10 \% \beta$-mercaptoethanol and $0.01 \%$ bromophenol blue), boiled in a heating block for 5 min and then loaded onto a $10 \%$ polyacrylamide gel for electrophoretic separation. Subsequently, the gels were either stained with EZBlue following the manufacturer's instructions or the proteins were transferred to a PROTRAN nitrocellulose membrane (Whatman, Dassel, Germany). The membranes were blocked with phosphate-buffered saline supplemented with 0.1\% Tween-20 (PBST) and 5\% nonfat dry milk (Bio-Rad, Munich, Germany). Following blocking, the membranes were incubated with primary antibodies at the adequate dilution in PBST supplemented with $5 \%$ milk overnight at $4{ }^{\circ} \mathrm{C}$. Incubation with peroxidase-labeled secondary rat or mouse antibodies that bind to all primary antibodies was conducted at room temperature for $1 \mathrm{~h}$, followed by extensive washing with PBST. Bands were visualized after addition of the Amersham ECL advanced Western blotting detection kit (GE Healthcare, Buckinghamshire, U.K.) with the aid of a Fujifilm LAS-3000 Documentation System (Fujifilm, Tokyo, Japan).

Antibody Microarray Printing. For assaying quantitatively the performance parameters of complex protein extracts on antibody microarrays, 38 mouse and rabbit antibodies from different sources were spotted six times on epoxy-coated slides (Nexterion-E; Schott, Jena, Germany) using the contact printer MicroGrid-2 (BioRobotics, Cambridge, U.K.) and SMP3B pins (Telechem, Sunnyvale, CA) at a humidity of $40-45 \%$. The printing buffer was composed of $0.1 \mathrm{M}$ borate buffer, $\mathrm{pH} 9.0$, containing $0.006 \%$ Igepal CA-630, $0.05 \%$ sodium azide, $1 \%$ Trehalose and $1 \mathrm{mg} / \mathrm{mL}$ of the respective antibody. After printing, the slides were allowed to equilibrate at a humidity of $40-45 \%$ overnight and then stored in dry and dark conditions at $4{ }^{\circ} \mathrm{C}$.

Protein Labeling with Fluorescent Dyes. The NHS-esters of the dyes DY-649 and DY-549 (Dyomics, Jena, Germany) were used for labeling extracted proteins. Samples from human pancreatic cancer tissue or cell culture were adjusted to a protein concentration of 4 or $2 \mathrm{mg} / \mathrm{mL}$, respectively. The labeling reaction occurred in the dark in $0.1 \mathrm{M}$ carbonate buffer, $\mathrm{pH} 8.5$, with a dye/protein ratio of $30: 1 \mathrm{~mol}$ at $4{ }^{\circ} \mathrm{C}$ for $2 \mathrm{~h}$. An amount of $400 \mu \mathrm{g}$ of protein was mixed with $200 \mathrm{nmol}$ dye in a final volume of $200 \mu \mathrm{L}$ of buffer. An average molecular weight of 60000 Da was assumed for cellular proteins to calculate the above dye/protein ratio. Unreacted dye was removed using Zeba Spin Columns (Thermo Scientific) with buffer exchange to PBS according to the manufacturer's recommendation. After labeling, the measured dye/protein ratio was within a range of $0.75-1.25$ in all experiments. Labeled samples were stored at $-20^{\circ} \mathrm{C}$ until use.

Incubation, Scanning, and Image Processing of the Microarrays. All subsequent procedures were performed in the dark. Before incubation with labeled proteins, slides were washed 4 times (5 min each) with PBST. Following washing, the slides were blocked with $5 \%$ nonfat dry milk in PBST for $3 \mathrm{~h}$ at room temperature. Blocked slides were incubated with $75 \mu$ g each of DY-549- and DY-649-labeled protein diluted in 5 mL of PBST, $5 \%$ milk at $4{ }^{\circ} \mathrm{C}$ overnight. The slides were then washed 4 times (5 min each) with PBST, rinsed with deionized water and dried in a ventilated oven at $37^{\circ} \mathrm{C}$. Scanning of the slides was performed using a ScanArray-4000XL (Perkin-Elmer, Waltham, MA) at constant laser power and PMT. The images were analyzed with the software GenePix Pro 6.0 (Molecular Devices, Sunnyvale, CA). The detection limit was similar to that reported previously. ${ }^{10,11}$

Data Analysis. Results are presented as means $( \pm \mathrm{SD})$ unless otherwise specified. Microarray data were analyzed without normalization using the software Acuity 4.0 (Molecular Devices, Sunnyvale, CA). Since each sample was labeled with both DY549 and DY-649, the sum of medians was applied for comparing target signal intensities among tested buffers. The sum of medians is the sum of the medians of the pixel intensities at each wavelength, with the median background pixel intensity at each wavelength subtracted (GenePix Pro 6.0 analysis software tutorial, http://www.moleculardevices.com). The median of feature signal and background intensity was used to determine quality control measures like signal-to-noise ratio (SNR) and signal intensity thresholds. The SNR in both color channels was calculated as the mean (from three replicate arrays) of median signal intensity (background subtracted) divided by the standard deviation of the median local background intensities. Cutoff values of (mean $-1 \times \mathrm{SD}$ ) for SNR and median signal intensity were used to threshold features in compliance with the quality measures. Statistical significance was determined with SSPS 16.0 for Windows (SPSS, Inc., Chicago, IL) using one-way analysis of variance (ANOVA) with post hoc LSD test for multiple analyses between groups. Results with $p$-values less than 0.05 were considered to be significant.

\section{Results and Discussion}

Like other proteomic methodologies, antibody microarrays make use of samples from different sources, ranging from material that requires minimal to no protein extraction, such as body fluids, to those that entail protein isolation step(s), such as cells in culture or tissue samples. As opposed to mass spectrometry analyses, the performance of antibody microarrays is strongly influenced by factors like protein structure (e.g., native or denatured), which is affected by the isolation procedure. In consequence, the protein extraction process is more critical than for methods which work with denatured molecules. However, in turn, this sensitivity permits studies on protein isoforms, for example, which are of particular interest for many pharmaceutical purposes. Therefore, we were interested in a process that preserves proteins in a near-native state.

Another major obstacle for establishing an appropriate protein extraction procedure was the requirement for compatibility of the resulting extracts with subsequent analytical procedures. Interfering substances that may affect protein quality or essential experimental processes, such as protein labeling, which is strongly influenced by the presence of nucleic acids, reductants or quenchers, for example, could influence the outcome of studies significantly. Also, complicated protocols have an intrinsically higher chance of introducing experimental bias. Thus, we aimed at a protocol, which consisted of a single incubation subsequent to the physical disruption of the cellular material.

Our criteria for formulating the basic composition of the protein extraction buffer were based on several factors: extraction should occur in a single step under mild, nondenaturing conditions; there should be no compounds that contain primary amines or thiols, which inhibit the fluorescence labeling; the effect of interfering biomolecules, such as nucleic acids should be minimized; and most importantly, solubiliza- 


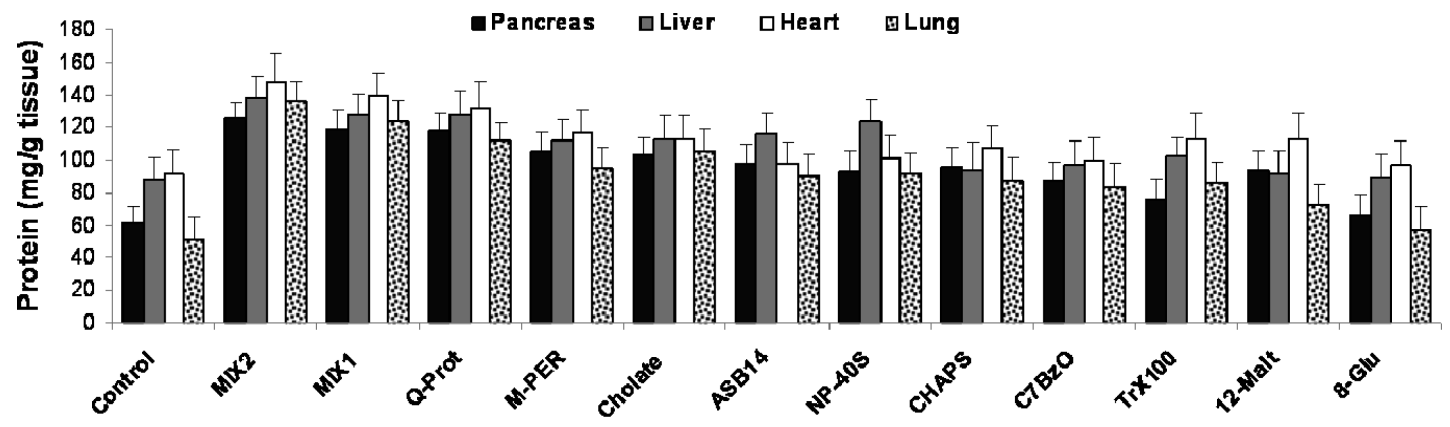

Figure 1. Protein yield with different detergents. The protein concentrations of extracts from pancreas, liver, heart and lung tissues are shown, which were obtained with the indicated buffer compositions. The average of three independent measurements is shown.

tion of compartmentalized proteins should occur in an effective and reproducible manner. As a consequence, substances commonly used for protein extraction, such as Tris-base, dithiothreitol and sodium dodecylsulfate (SDS), were excluded. As an alternative to Tris-base, we tested several other buffering substances like 4-(2-hydroxyethyl)-1-piperazinepropanesulfonic acid (EPPS), 4-( $N$-morpholino)butanesulfonic acid (MOBS), 1,4piperazinediethanesulfonic acid (PIPES) and 4-(2-hydroxyethyl)piperazine-1-ethanesulfonic acid (HEPES). This group of buffers, also known as Good's buffers, ${ }^{21}$ are characterized by their high compatibility with biological analysis, good solubility in water, minor salt effects and minimal interference with biological functions. We found HEPES buffer to be the most suitable milieu for protein extraction. To block interference from nucleic acids, we added the enzyme Benzonase to the buffer, which destroys these macromolecules. To avoid protein degradation by the cellular protein-digesting machinery, a commercially available cocktail of protease and phosphatase inhibitors was added, and the temperature was kept at $0{ }^{\circ} \mathrm{C}$ or less throughout analysis. The presence of $20 \%$ glycerol prevented freezing of the solution.

Most critical to a successful isolation, however, were the detergents used in the process. They were selected from groups of nonionic, ionic and zwitterionic nondenaturing detergents. In addition to studying the effect of individual detergents, we formulated two detergent mixtures to assess the combined effect of these detergents on cellular protein recovery. The detergent mixes were composed of up to four detergents that are of entirely different chemical structure and represent a broad spectrum of detergents generally used for protein extraction from biological specimens. The chemical classes to which the chosen detergents belong are polymeric phenylethylene glycols, long-chain alkyl amidosulfobetaines, cholic acid derivatives and long-chain alkylglycosides. A mixture of chemical structures should be superior to overcome the steric obstacle posed by the lipids engulfing protein geometry. Additionally, different classes of detergents have demonstrated their preference for isolating proteins from particular cellular compartments. ${ }^{14,22}$ A combination may add more power to isolating proteins from the various cellular organelles. After preliminary tests (data not shown), we ended up with two mixtures that exhibited good results.

The first detergent mixture (Mixl) has a composition somewhat similar to that routinely used for two-dimensional gel electrophoresis ${ }^{12}$ with chaotropes like urea, thiourea and ampholytes being excluded. The presence of these substances is necessary in electrophoresis in order to prevent the precipitation of protein during the gel separation. They are not required for antibody array assays, however. On the contrary, since chaotropes are denaturant to proteins, their absence improves protein functionality. The second detergent mixture (Mix2) is composed of detergents that are mimicking in their chemical structure those of radioimmunoprecipitation assay (RIPA) buffer. In this mixture, Nonidet P-40 substitute, sodium cholate and ASB-40 were the substitutes for NP-40, sodium deoxycholate and SDS in RIPA buffer, respectively. However, both mixtures contained long-chain alkyl-glycosides, since they have been reported to have extraction capacity to integral membrane proteins. ${ }^{5,12}$

For a comparative evaluation of the overall procedure, we also analyzed several commercial buffer systems. In an initial examination, several of them exhibited a limited extraction capacity or an apparent bias for particular protein types or compartmental protein fractions (data not shown). From all buffers tested, the Q-Proteome Mammalian Protein Prep (QProt) kit of Qiagen and Mammalian Protein Extraction Reagent (M-PER; Thermo Scientific) were chosen as controls, since they showed the best results and are also meant to extract proteins at nondenaturing conditions.

Protein Levels Extracted from Rat Tissues and Cell Cultures. Our first experiments were aimed at evaluating the capacity of various detergents, either individually or in the two mixtures, to extract cellular proteins from solid (heart and lung) and soft (liver and pancreas) rat tissues. Tissue homogenization was performed using four mechanical approaches: homogenization with Ultra Turrax, Dounce and Potter-Elvehjem homogenizers or pulverization with mortar and pestle after freezing the tissue in liquid nitrogen. Generally, we found that pulverization of samples worked best for hard tissues like heart and lung tissue, while homogenization using a Potter-Elvehjem homogenizer gave the most satisfactory results for liver and pancreatic tissue (data not shown). However, since the differences were minor, we used the pulverization method for all tissue samples in order to minimize sources of variation and for the purpose of uniformity.

The efficiency of protein extraction was evaluated by measuring the amount of protein resulting from each preparation per gram of tissue (Figure 1) and comparing to a buffer devoid of detergent (control) and the commercial extraction systems. While there were some apparent differences between the four tested tissues, the overall tendency was the same across the experiment. Mix2, Mix1 and Q-Prot performed best, sodium cholate was the most effective individual detergent on all types of tissues studied, the polymeric and zwitterionic detergents showed moderate extraction efficiency, and 8-Glu delivered the least extraction potential.

Next, we studied the quantity and functionality of proteins extracted from human BxPC-3 and SU.86.86 cell lines. For both, 

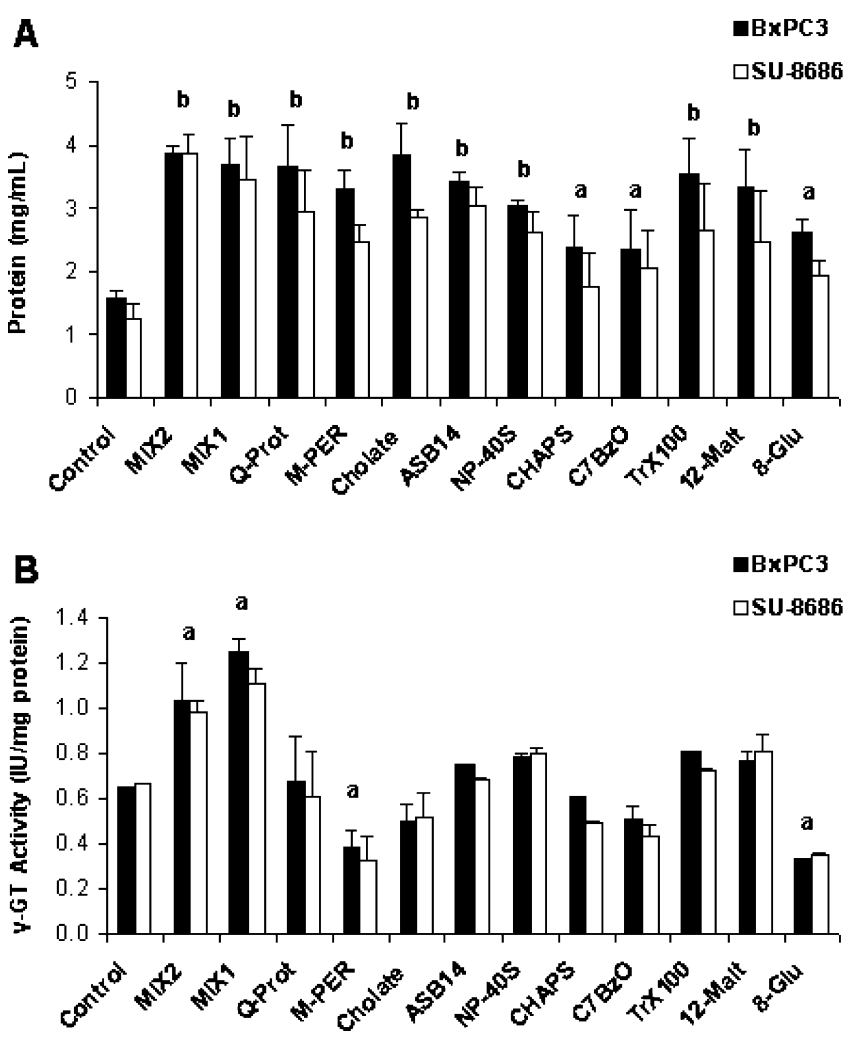

Figure 2. Protein extraction on BxPC-3 and SU.86.86 cell line lysates. The overall protein yield (A) and the specific $\gamma$-GT activity (B) are shown, which were obtained with three buffers. The average value from three independent measurements is shown. The significance compared to control buffer is $p<0.01$ (a) and $p$ $<0.001$ (b), respectively.

the highest protein yield was obtained upon extraction with Mix1 and Mix2, followed by sodium cholate, ASB14 and the commercial Q-Prot buffer (Figure 2A). While the differences were not big between the best performing buffers, they were consistent throughout and differences were substantial between the best and worst performing buffers. The two detergent mixtures succeeded in preserving the enzymatic activity of $\gamma$-glutamyltranspeptidase $(\gamma$-GT) at the highest level (Figure 2B). Since a lower concentration of each individual detergent is used in the mixtures compared to buffers with just one detergent, the detergents may exert a less negative effect on protein integrity, while nevertheless in their sum guarantee effective extraction. Alternatively, the high activity could be explained by an enrichment of the membrane protein fraction upon extraction with the detergent cocktails; $\gamma$-GT is a membrane-associated enzyme.

Detergent Effects on Protein Extracted from Human Pancreatic Tissue. On the basis of the results with rat tissues and human cell lines, we performed studies with the three best buffer systems on 12 human pancreatic cancer tissues, which were randomly selected from a larger set of samples. Mix2 resulted in a significantly higher protein yield compared to Q-Prot and Mixl (Figure 3A). Analysis of the enzymatic activity showed a different pattern, however. Protein extracted with Mixl exhibited significantly higher enzymatic activities for $\gamma$-GT (membrane bound), GST (membrane bound and cytoplasmic) and LDH (cytoplasmic) as compared to the Q-Prot kit (Figure 3C). The lower enzymatic activity obtained by Mix2 extraction is probably a result of inhibitory effects of $8-\mathrm{Glu}$, which is
A

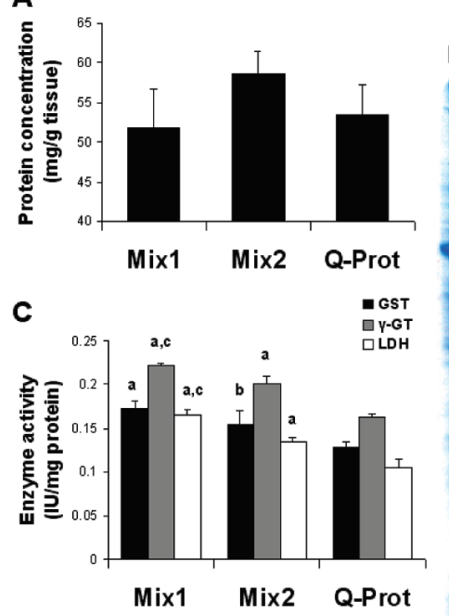

B

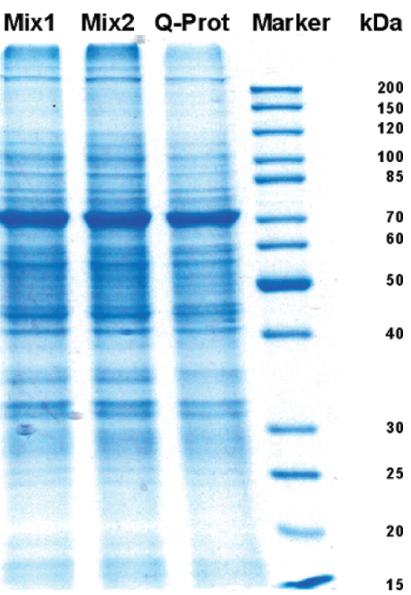

Figure 3. Quantitative and functional analysis of protein extracted with Mix1, Mix2 and Q-Prot, respectively, from a pool of 12 human pancreatic cancer tissues. The results are mean values $( \pm \mathrm{SD}$ ) of three experiments. (A) The protein yield is shown. (B) The three different protein extracts were run on a $10 \%$ acrylamide gel and stained with Coomassie blue. (C) Measurements of the specific enzymatic activities of GST, $\gamma$-GT and LDH. Significances are (a) $p<0.005$ vs Q-Prot, (b) $p<0.05$ vs Q-Prot and (c) $p<0.05$ vs Mix2.

present in this mixture. It has been shown that detergents with short $\left(\mathrm{C}_{7}-\mathrm{C}_{10}\right)$ hydrocarbon chain like octylglucoside are more inactivating than the corresponding detergents with an intermediary $\left(\mathrm{C}_{12}-\mathrm{C}_{14}\right)$ hydrocarbon chain like $\beta$-dodecylmaltoside..$^{23,24}$ Also in the cell culture experiments, the least $\gamma$-GT activity was found in protein extracted with 8-Glu (Figure 2B). We cannot comment on the reason for the lower enzymatic activity in extracts obtained with Q-Prot, since the composition of the buffer is not publicly known.

Effect of Extraction Buffers on Proteins from Cellular Organelles. To determine the efficiency of the extraction methods in recovering proteins from cellular organelles, we isolated proteins from human pancreatic cancer tissues followed by a qualitative and quantitative assessment of proteins by Western blot analysis and antibody microarrays. We looked at the following marker proteins: $\gamma$-GT [plasma membrane], flotillin [plasma membrane], caveolin [plasma membrane], $\beta$-actinin [cytoskeleton], TGN-46 [Golgi apparatus], calreticulin [endoplasmatic reticulum], catalase [peroxisome], cathepsin D [lysosome], lamin A [nucleus] and cytochrome C [mitochondria] (Figure 4). Overall, the results obtained by immunoblotting and microarray analysis, using the same antibodies, were in good agreement. With regard to protein yield, both Mixl and Mix2 showed a much better efficiency in extracting membrane-associated proteins compared to Q-Prot.

Plasma membrane is one of the richest sources of cellular proteins that are important with respect to disease and therapy. Around $30 \%$ of the human proteins are embedded in the membrane. ${ }^{25,26}$ Membrane proteins are involved in many important cellular events like cell signaling and signal transduction, transport, and cellular communication, besides others. It is well-documented that proteins of plasma membranes pose a complicating factor during proteomic analysis owing to the high hydrophobicity. ${ }^{27}$ We investigated the capacity of the detergent cocktails for isolating highly hydrophobic proteins and found superior performance, revealed by both Western blot 

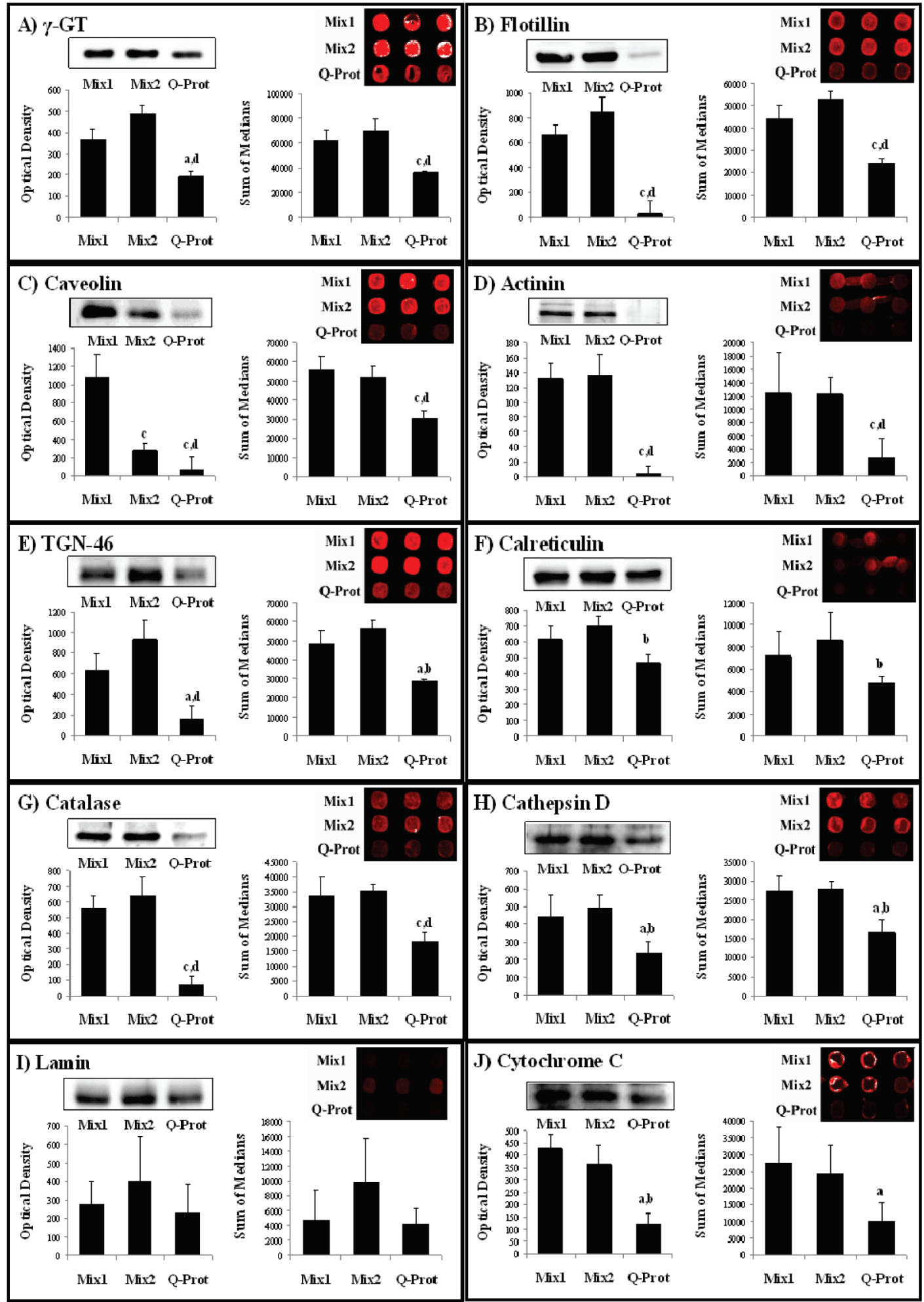

Figure 4. Western blot and antibody microarray results of cellular organelle marker proteins. In each panel, typical experimental results are shown. In addition, bar charts present the mean values of three separate experiments each; this equals 18 data points from microarray measurements. (A) $\gamma$-GT [plasma membrane], (B) flotillin [plasma membrane], (C) caveolin [plasma membrane], (D) $\beta$-actinin [cytoskeleton], (E) TGN-46 [Golgi apparatus], (F) calreticulin [endoplasmatic reticulum], (G) catalase [peroxisome], (H) cathepsin D [lysosome], (I) lamin A [nucleus] and (J) cytochrome C [mitochondria]. P-values: $<0.05$ vs Mix1 (a), $<0.05$ vs Mix2 (b), <0.005 vs Mix1 (c), and $<0.005$ vs Mix2 (d).

and antibody microarray analyses. Zwitterionic detergents and alkyl-glycoside have been suggested as better solubilizers of membrane proteins than polyethylene glycols like Triton X100 and NP-40. ${ }^{12}$ We found no significant difference in the extrac- tion efficiency between these detergent groups. However, in combination, they resulted in a much better yield of protein quantity and functional quality. The latter may be attributed to the lower concentration of each detergent individually. 


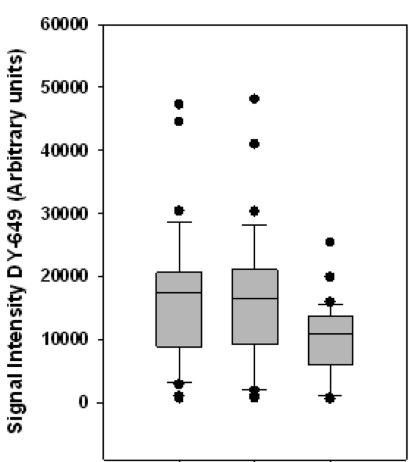

Mix1 Mix2 Q-Prot

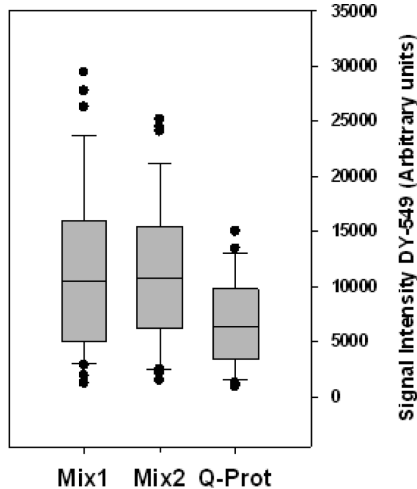

Mix1 Mix2 Q-Prot
Figure 5. Box plot of the signal intensities obtained with samples prepared with Mix1, Mix2 and Q-Prot, respectively, and labeled with both red (DY-649) and green (DY-549) fluorescence dye. The values represent the means of three independent measurements.

Furthermore, cytoskeletal proteins are categorized among the hardest to solubilize. It has been shown that these proteins resist Triton X100 disruption. ${ }^{28} \mathrm{He}$ et al. recently reported a solubilization of cytoskeletal proteins using ASB-14. ${ }^{29}$ Indeed, in our experiments, we were able to see apparent signals on the arrays and bands in the Western blots with both mixtures, each of which contained ASB-14.

Of the plasma membrane proteins, only caveolin showed a different result insofar as it was the only protein which could not be extracted similarly well with Mix2 as with Mixl. However, both buffers exhibited good solubilization of the cytoskeletal protein $\beta$-actinin and the other proteins from cellular organelles. Mix2 was almost always slightly better than Mix1 in protein extraction, although not significantly. Lamin A (nuclear envelope; Figure 4I) exhibited the smallest difference between the three buffer systems. However, microarray data for other nuclear proteins, which were not particularly confirmed by Western blot analysis, showed a higher recovery for all of these with Mix1 and Mix2 as compared to Q-Prot (Supporting Information).

Quality Control on Antibody Microarrays. We applied stringent quality control criteria to check the performance of the extracts on antibody microarrays. Quality control measures like signal intensity, signal-to-noise ratio (SNR) and dye effect were used to check the compatibility of tested buffers with the antibody microarrays. Overall, the detection limit of the antibody microarrays was similar to that reported previously. ${ }^{10,11}$ For the signal-to-noise ratio, the threshold values (mean value $-1 \times \mathrm{SD}$ ) were 2.0 and 3.2 for DY-649 and DY-549, respectively. Similarly, only signal intensities (background subtracted) ex- ceeding a cutoff (mean $-1 \times$ SD) value of 4243 and 3083 arbitrary units for the red and green detection channels, respectively, were considered adequate. Pancreatic cancer samples were labeled with the dyes DY-649 (red) and DY-549 (green), respectively. Two differently labeled protein preparations were then mixed and incubated on microarrays made of 38 antibodies, each spotted in six replicates. From the results, we calculated the percentage of microarray features that exhibited a value above the respective threshold. Uniform conditions were applied throughout the analysis with both fluorescent dyes. Protein extracts isolated with Mix1 and Mix2 produced the best results on the arrays followed by Q-Prot in terms of signal intensity (Figure 5) and overall SNR (Table 1). Q-Prot extracted fewer proteins that produced signal intensities which exceeded the threshold limit for both channels (Table 1). This result is important when detecting low-abundance proteins. Such proteins may go undetected not as a result of limited sensitivity of the array assay, but rather because of the inefficiency of the extraction buffer. In addition, the two dyes used in the analysis behaved very similarly in the labeling of samples obtained with both Mixl and Mix2, while clear differences were observed with Q-Prot. As documented by this result, the unknown composition of commercial buffers could pose a serious limitation in condition optimization and detecting potential sources of error. All amines in Mix1 and Mix2 are tertiary amines, for example, and should therefore not affect the dye NHS-esters, which only react with primary amines.

Since each sample was labeled with both dyes, a similar pattern of signal intensities for target proteins is expected at both channels. This was the case with both Mix1 and Mix2. The results demonstrated a high correlation between the red and green channel detection for all 38 probes (Figure 6). Q-Prot, on the other hand, exhibited more variations, although identical conditions had been used in the experiments. This difference complicates two-color measurements considerably. As already mentioned above, we cannot comment on possible reasons for this effect for the lack of information about the composition of the commercial product. However, the very good correlation observed for protein samples extracted with Mix1 and Mix2 indicated that they have no significant effect on the labeling process.

\section{Conclusions}

Two buffer cocktails were established that demonstrated their compatibility with and applicability to proteomic analysis based on antibody microarrays and were superior to the best systems available to date. We currently use the cocktails for routine analyses of cell culture and tissue samples on complex antibody microarrays, made from a panel of 810 different

Table 1. Percent of Antibody Probes (Total Number is 38, Each Spotted in Six Replicates) That Showed Acceptable Quality in Terms of Signal Intensities and Signal-to-Noise Ratio ${ }^{a}$

\begin{tabular}{|c|c|c|c|c|}
\hline & \multicolumn{2}{|c|}{ signal-to-noise ratio } & \multicolumn{2}{|c|}{ signal intensity (minus background) } \\
\hline Mix1 & $93.86 \% \pm 4.02 \%$ & $90.35 \% \pm 3.04 \%$ & $85.96 \% \pm 4.02 \%$ & $89.47 \% \pm 0.00 \%$ \\
\hline \multirow[t]{2}{*}{ Q-Prot } & $94.74 \% \pm 2.63 \%$ & $82.46 \% \pm 6.62 \%$ & $79.82 \% \pm 1.52 \%$ & $77.19 \% \pm 4.02 \%$ \\
\hline & $\begin{array}{l}\text { Threshold defi } \\
\text { mean value - } \\
2.0\end{array}$ & 3.2 & $\begin{array}{l}\text { Threshold defi } \\
\text { mean value - } \\
4243 \text { units }\end{array}$ & 3083 units \\
\hline
\end{tabular}

${ }^{a}$ The definition and actual values of the thresholds are given. The units for signal intensity are arbitrary values. 

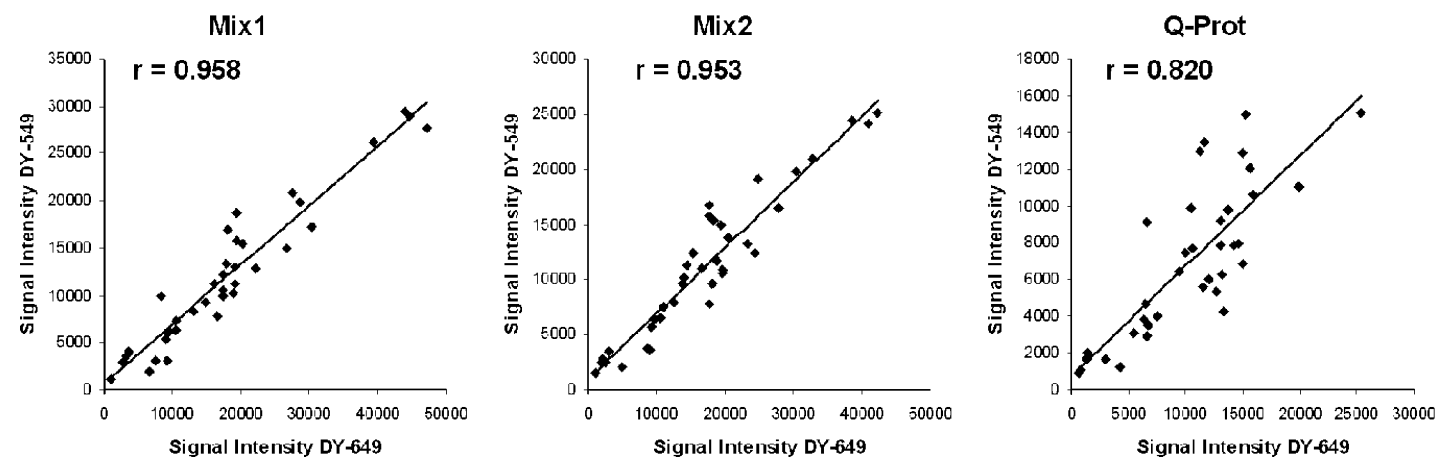

Figure 6. Correlation and Pearson correlation coefficient $(r)$ between DY-649 and DY-549 labeled proteins extracted with Mix1, Mix2 and Q-Prot $(n=38)$.
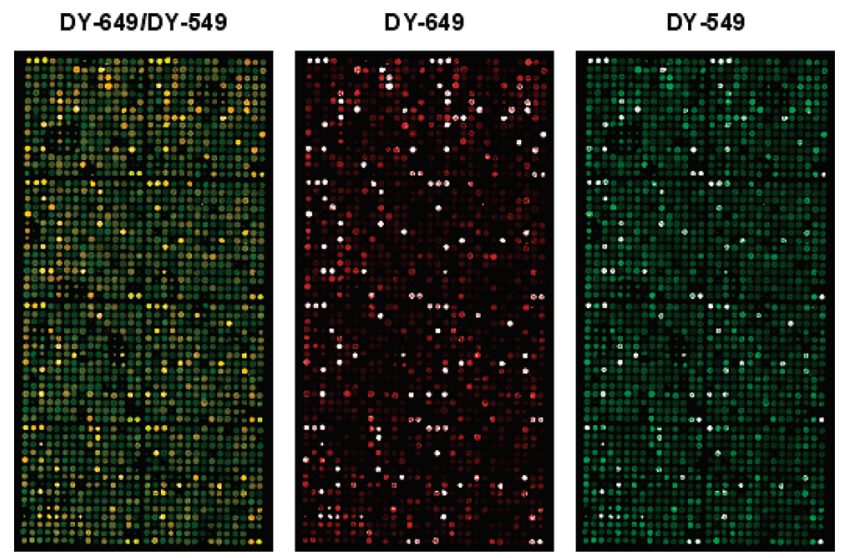

Figure 7. Result from a routine two-color expression measurement on a complex antibody microarray. Protein was extracted from BxPC-3 cell line with Mix2, labeled with DY-649 or DY-549, and incubated as described in Material and Methods on a complex microarray made of 810 different antibodies. This antibody panel targets proteins, whose genes had been identified to be differentially transcribed on the RNA-level in cancer versus normal tissue. Both the images of the individual detection channels and the combined image are shown.

antibodies, each spotted in duplicate. The studies aim at the elucidation of biological variations of cancer-relevant proteins. As part of these large-scale studies, also the identical samples are labeled with the two dyes and analyzed in competitive incubations on one microarray for the purpose of a continuous quality assessment (e.g., Figure 7), again demonstrating their assay compatibility. Both buffers are composed of detergent combinations that include at least one polymeric phenylethylene glycol, a long-chain amidosulfobetaine, cholate and a long-chain alkylglycoside. Their use leads to a reproducible and simple extraction of protein from tissues. While Mix2 has a higher overall yield judging from immunoblot experiments, Mixl is slightly superior with regard to functional aspects. One should keep in mind, however, that functional aspects could well be affected by the addition of fluorophore labels. We aim at analyzing not only expression differences with regard to protein levels but also conformational variations, such as protein isoforms. Consequently, isolation of proteins in a structurally relevant form is critical. Nevertheless, factors such as chemical derivatization could influence the results considerably. Overall, the protein extracts resulting from incubations with Mixl and Mix2 exhibited a good representation of membrane-associated proteins and worked with high efficiency. The latter is especially important for analyzing small clinical samples. While established for antibody array applica- tions, the high agreement of the array data and the Western blot analyses indicates that the buffers could also be useful for other types of proteomic studies.

Acknowledgment. We are grateful to Dr. Werner Nicklas for provision of the rat tissues. This work was financially supported by the EU-funded projects ProteomeBinders and MolDiagPaCa. M.S.S.A. received a long-term fellowship from the Deutscher Akademischer Austausch Dienst (DAAD).

Supporting Information Available: Figure 1S, antibody microarray results of the other 28 proteins for which no Western blot experiments were conducted. The values are arbitrary units (AU) of the mean of the sum of medians for signal intensities from three arrays. This material is available free of charge via the Internet at http://pubs.acs.org.

\section{References}

(1) Alhamdani, M. S.; Schröder, C.; Hoheisel, J. D. Oncoproteomic profiling with antibody microarrays. Genome Med. 2009, 1 (7), 68.

(2) Haab, B. B. Methods and applications of antibody microarrays in cancer research. Proteomics 2003, 3 (11), 2116-22.

(3) Lv, L. L.; Liu, B. C. High-throughput antibody microarrays for quantitative proteomic analysis. Expert Rev. Proteomics 2007, 4 (4), 505-13.

(4) Wingren, C.; Borrebaeck, C. A. Antibody-based microarrays. Methods Mol. Biol. 2009, 509, 57-84.

(5) Leimgruber, R., Extraction and Solubilization of Proteins for Proteomic Studies. In The Proteomics Protocols Handbook; Walker, J. M., Ed.; Humana Press: Totowa, NJ, 2005; pp 1-18.

(6) Dexlin, L.; Ingvarsson, J.; Frendeus, B.; Borrebaeck, C. A.; Wingren, C. Design of recombinant antibody microarrays for cell surface membrane proteomics. J. Proteome Res. 2008, 7 (1), 319-27.

(7) Wong, L. L.; Chang, C. F.; Koay, E. S.; Zhang, D. Tyrosine phosphorylation of PP2A is regulated by HER-2 signalling and correlates with breast cancer progression. Int. J. Oncol. 2009, 34 (5), 1291-301.

(8) Hao, Y.; Yu, Y.; Wang, L.; Yan, M.; Ji, J.; Qu, Y.; Zhang, J.; Liu, B.; Zhu, Z. IPO-38 is identified as a novel serum biomarker of gastric cancer based on clinical proteomics technology. J. Proteome Res. 2008, 7 (9), 3668-77.

(9) Kusnezow, W.; Jacob, A.; Walijew, A.; Diehl, F.; Hoheisel, J. D. Antibody microarrays: an evaluation of production parameters. Proteomics 2003, 3 (3), 254-64.

(10) Kusnezow, W.; Syagailo, Y. V.; Ruffer, S.; Baudenstiel, N.; Gauer, C.; Hoheisel, J. D.; Wild, D.; Goychuk, I. Optimal design of microarray immunoassays to compensate for kinetic limitations: theory and experiment. Mol. Cell. Proteomics 2006, 5 (9), 168196.

(11) Kusnezow, W.; Banzon, V.; Schröder, C.; Schaal, R.; Hoheisel, J. D.; Ruffer, S.; Luft, P.; Duschl, A.; Syagailo, Y. V. Antibody microarraybased profiling of complex specimens: systematic evaluation of labeling strategies. Proteomics 2007, 7 (11), 1786-99.

(12) Rabilloud, T. Detergents and chaotropes for protein solubilization before two-dimensional electrophoresis. Methods Mol. Biol. 2009, 528, 259-67. 
(13) McCarthy, F. M.; Cooksey, A. M.; Burgess, S. C. Sequential detergent extraction prior to mass spectrometry analysis. Methods Mol. Biol. 2009, 528, 110-8.

(14) Börner, A.; Warnken, U.; Schnolzer, M.; Hagen, J.; Giese, N.; Bauer, A.; Hoheisel, J. Subcellular protein extraction from human pancreatic cancer tissues. BioTechniques 2009, 46 (4), 297-304.

(15) Simpson, R., Proteins and Proteomics: A Laboratory Manual Cold Spring Harbor Laboratory Press: Cold Spring Harbor, NY, 2003.

(16) Rabilloud, T.; Blisnick, T.; Heller, M.; Luche, S.; Aebersold, R.; Lunardi, J.; Braun-Breton, C. Analysis of membrane proteins by two-dimensional electrophoresis: comparison of the proteins extracted from normal or Plasmodium falciparum-infected erythrocyte ghosts. Electrophoresis 1999, 20 (18), 3603-10.

(17) Chevallet, M.; Santoni, V.; Poinas, A.; Rouquie, D.; Fuchs, A.; Kieffer, S.; Rossignol, M.; Lunardi, J.; Garin, J.; Rabilloud, T. New zwitterionic detergents improve the analysis of membrane proteins by two-dimensional electrophoresis. Electrophoresis 1998, 19 (11), 1901-9.

(18) Shaw, L. M.; London, J. W.; Fetterolf, D.; Garfinkel, D. GammaGlutamyltransferase: kinetic properties and assay conditions when gamma-glutamyl-4-nitroanilide and its 3-carboxy derivative are used as donor substrates. Clin. Chem. 1977, 23 (1), 79-85.

(19) Habig, W. H.; Pabst, M. J.; Jakoby, W. B. Glutathione S-transferases. The first enzymatic step in mercapturic acid formation. J. Biol. Chem. 1974, 249 (22), 7130-9.

(20) Babson, A. L.; Babson, S. R. Kinetic colorimetric measurement of serum lactate dehydrogenase activity. Clin. Chem. 1973, 19 (7) 766-9.
(21) Good, N. E.; Winget, G. D.; Winter, W.; Connolly, T. N.; Izawa, S.; Singh, R. M. Hydrogen ion buffers for biological research. Biochemistry 1966, 5 (2), 467-77.

(22) Ramsby, M. L.; Makowski, G. S. Differential detergent fractionation of eukaryotic cells. Analysis by two-dimensional gel electrophoresis. Methods Mol. Biol. 1999, 112, 53-66.

(23) le Maire, M.; Champeil, P.; Moller, J. V. Interaction of membrane proteins and lipids with solubilizing detergents. Biochim. Biophys. Acta 2000, 1508 (1-2), 86-111.

(24) Lund, S.; Orlowski, S.; de Foresta, B.; Champeil, P.; le Maire, M.; Moller, J. V. Detergent structure and associated lipid as determinants in the stabilization of solubilized Ca2+-ATPase from sarcoplasmic reticulum. J. Biol. Chem. 1989, 264 (9), 4907-15.

(25) Wallin, E.; von Heijne, G. Genome-wide analysis of integral membrane proteins from eubacterial, archaean, and eukaryotic organisms. Protein Sci. 1998, 7 (4), 1029-38.

(26) Babiychuk, E. B.; Draeger, A. Biochemical characterization of detergent-resistant membranes: a systematic approach. Biochem. J. 2006, 397 (3), 407-16.

(27) Tan, S.; Tan, H. T.; Chung, M. C. Membrane proteins and membrane proteomics. Proteomics 2008, 8 (19), 3924-32.

(28) Luna, E. J.; Hitt, A. L. Cytoskeleton--plasma membrane interactions. Science 1992, 258 (5084), 955-64.

(29) He, J.; Liu, Y.; He, S.; Wang, Q.; Pu, H.; Ji, J. Proteomic analysis of a membrane skeleton fraction from human liver. J. Proteome Res. 2007, 6 (9), 3509-18.

PR900844Q 